\title{
- Case Report
}

\section{The cysticercosis in muscles is one of the causes of lumps in human}

\author{
BK Rai, Shashikar \\ Dept of GP\&EM, B.P Koirala Institute of Health Sciences, Dharan
}

\begin{abstract}
Introduction: Cysticercosis is commonly encountered in this part of the world. It can manifest in many different form. Objective: To report a case of cysticercosis which presented with a lump in axilla. Case: A 36 year old man from Dharan presented with the chief complaint of lump in the right axilla for 9 months and two lumps in left axilla for 3 months. The onset of lump was insidious and gradually progressive in nature. The examination revealed a single mass in the medial surface of right upper arm, approximately $3 \times 3 \mathrm{~cm}$ in size, not fixed with the skin, slightly movable and mildly tender and another two masses around $1 \times 2 \mathrm{~cm}$ in left axilla with similar characteristics as the right one. The ultrasonography showed heterogenous lesion measuring $27 \mathrm{~mm} \times 18 \mathrm{~mm}$ in medial aspect of right upper arm and two similar lesions measuring $14.5 \times 11 \mathrm{~mm}$ and $11 \mathrm{x} 10 \mathrm{~mm}$ in left axilla suggesting "Cysticercosis". The lumps were surgically removed successfully. Conclusion: Cysticercosis is one of the causes of lump in axillay region. It can be diagnosed ultrasonographically and treated by surgical removal.
\end{abstract}

Key words: axilla, cysticercosis, ultrasonography

\section{Introduction}

Cysticercosis (tapeworm infection) is an increasingly common medical problem in worldwide. An estimated 50-100 million people are infested with cysticercosis worldwide. This is probably an underestimate since many infections go undiagnosed. Cysticercosis is caused by the larval stage of Taenia solium, the pork tapeworm. The Clinical syndrome caused by this parasite is categorized as either neurocysticercosis (NCC) or extraneural cysticercosis (intestinal tapeworm, subcutaneous or muscular cysticerci infection which is one of the causes of lump in human beings.

Neurocysticercosis, which is probably the most common parasitic infestation of the CNS, has gained increased recognition in the last two decades because of the development of MRI and CT scanning. The

Address for correspondence

Dr. Bijendra Kumar Rai

Associate Professor, Dept of GP\&EM

B.P Koirala Institute of Health Sciences, Dharan

Email:Bijen001@gmail.com ultrasonogram is one of the diagnositic tool of cysticerci of subcutaneous and/or muscles. The cases can be treated medically or surgically. We report a surgically treated case of cysticercosis involving the axillary regions.

\section{Case history}

A 36 year old man from Dharan presented in General OPD of B.P. Koirala Institute of Health sciences on $23^{\text {rd }}$ January 2011 . He was a carpenter by profession and non-vegetarian. His chief complaints were lump in right axilla for 9 months and in the left axilla for 3 months. The onset was insidious, slowly progressive in nature and associated with occasional mild grade pain and discomfort while he was working.

On examination, there was a single mass in the medial surface of right upper arm, approximately $3 \times 3 \mathrm{~cm}$ in size, not fixed with skin, slightly movable and mildly tender and another two nodular masses around $1 \times 2 \mathrm{~cm}$ in left axillary region with similar in characteristics as the right one. 
The ultrasonography report showed "heterogenous lesions measuring $27 \mathrm{~mm} \times 18 \mathrm{~mm}$ in medial aspect of right upper arm and two similar lesions measuring $14.5 \times 11 \mathrm{~mm}$ and 11 x $10 \mathrm{~mm}$ in left axilla suggesting "Cysticercosis".

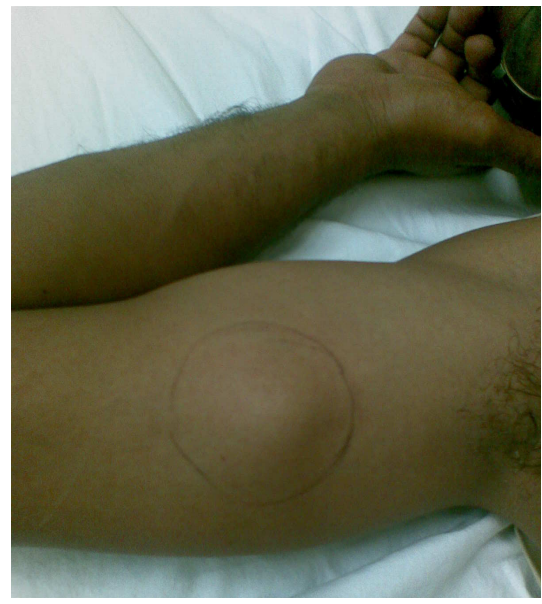

Fig 1: showing lump in medial Surface of right upper arm.

\section{Discussion}

The cause of human cysticercosis is the larval form of Taenia solium (pork tapeworm). Pigs and humans are T. solium reservoirs. Human can be infected by ingestion of the eggs or larvae from eating undercooked pork that contains viable cysticercosis larvae or from fecally contaminated food and water. Autoinfection as a result of the entry of eggs into stomach from the host's own feces due to contaminated hands is also possible. ${ }^{1}$ The incubation period ranges over months to ten years. ${ }^{2}$ Subcutaneous cysts are usually firm and mobile, nodules, occurring mainly on the trunk and extremities. ${ }^{3}$ Subcutaneous nodules are sometimes painful.

The Cysticerci can develop in any voluntary muscles in humans. Invasion of muscle by cysticerci can cause myositis with fever, eosinophilia and muscular pseudo hypertrophy leading to atrophy and fibrosis. In most cases, it is asymptomatic since the cysticerci die and become calcified. ${ }^{4}$

\section{Diagnostic test}

The traditional method of demonstration of T. sodium eggs in the stool samples diagnose only taeniasis. The immunoblot assay, cysticercosis- specific antibodies can react with structure glycoprotein antigen from the larval cysts of T. solium. Therefore, the serum samples from patients with other microbial infection do not react with any of the T. solium derived antigen. The tests are so far $100 \%$ specific and have higher sensitivity than any other immunoassays system. ${ }^{5} \mathrm{CT}$ scan shows both calcified and uncalcified cysts, as well as distinguishing active and inactive cysts. Howevere, the USG is the best diagnostic method for subcutaneous and muscles cysticerci where there is no CT and MRI facilities. ${ }^{6,7}$

\section{Treatment}

Treatment recommendations for subcutaneous and muscular cysticecosis include surgery, praziquantal and albendazole. Marshall Hightower's and his colleagues recently demonstrated that a recombinant ionosphere vaccine against $\mathrm{T}$. sodium is highly effective. Protection was $100 \%$ in the field and laboratory. Our case presented with lumps in axillary regions, which has not been reported yet. The case was diagnosed with USG-B scan and treated by surgically removal of the cysts.

\section{Conclusion}

The cysticercosis can present as lump in axillary region and upper arm. It can be diagnosed ultrasonographically and treated by surgically removal.

\section{References}

1. Southwick, Frederick (2007-12-10). "Chapter 12 Parasite Infections". Infectious diseases: A Clinical Short Course, $2^{\text {nd }}$ Ed...McGraw-Hill Medical Publishing Division. (2007) Pp. 313 314. ISBN 0071477225.

2. "Neurocysticercosis" Emerging Neurological Infections edited Power, $\mathrm{C}$ and Johnson RT. Taylor and Francis Group, 2005.261-287.

3. Workman PD. "Subcutaneous cysticercosis" J Am Aced Dermatology. 1991 (2 Pt 2):409-14.

4. MarcelEK, John DT, Trotsky WA. Medical Parasitological Eight edition. Pennsylvania: Saunders, 1999.

5. http:/www.cdc.gov/ncidod/parasites/ cysticercosis/factsht_cysticercosis.htm.

6. J. Clin.Ultrasound 2001 Oct: 29(8):472-5 Main NB, Kalra N, Jain M. Sindhu R.

7. Marshall Hightower's and his colleagues (Into J Parasite 2003; 33:1207-17

8. White AC Jr, Robinson P, Kuhn R. Taenia solium cysticercosis: host- parasite interactions and the immune response. Chem Immunol. 1997; 66:209-30.

9. Kraft R. Cysticercosis: an emerging parasitic disease. Am Fam Physician. Jul 1 2007;76(1):91-6. 